\title{
Identification of Physical Characteristic of Composite Materials Produced by Additive Technology from Perspective of Selected Mechanical Properties
}

\author{
M. SÁGA*, F. BÁrnik, M. VašKo, M. Handrik and P. Kopas \\ Department of Applied Mechanics, Faculty of Mechanical Engineering, University of Žilina, \\ Univerzitná 8215/1, 01026 Žilina, Slovak Republic \\ Doi: $10.12693 /$ APhysPolA.138.249 \\ *e-mail: milan.saga@fstroj.uniza.sk
}

\begin{abstract}
The aim of the paper is to present targeted experimental research of selected physical properties of composite structures produced by additive technology. The purpose is to identify optimum mechanical parameters, especially from the point of view of ultimate limit stress or long-term operational functionality. The focus is the experimental measurement on samples of polymer composite based on Onyx produced by additive technology. Onyx is a modern material based on remarkably strong nylon in combination with carbon microfibers. Its physical properties are significantly better than conventional polymers produced by 3D printing. Onyx is stronger, harder and thanks to carbon microfibers in the matrix, is more suitable for the production of components loaded by multiaxial stress in terms of mechanical properties. Standardized test samples were generated by 3D printing for the purpose of experimental measurements. Two types of 3D printing structure were used for the samples - fibers embedded at angle of $\pm 45^{\circ}$ and in the second case at angle of $0^{\circ}$ and $90^{\circ}$. Tensile tests were performed to identify the necessary physical properties. The influence of the composite structure shape on mechanical characteristics such as tensile strength limit, Young's modulus, Poisson's number and relative strain was studied. The mutual comparison of the measurement results is clearly processed in tabular and in graphical form. The comparative study showed a significant influence of fiber orientation, the number of layers and different types of internal structures on the investigated physical characteristic. The primary object of the presented experimental measurements is the analysis of the influence of different 3D printing variants on selected physical characteristic. The obtained results will be used to define inputs for algorithmization and simulation of computational models by FEM application with subsequent optimization of structural parameters in the next research of authors.
\end{abstract}

topics: physical characteristics, composite structure, additive technology, Onyx

\section{Introduction}

Composite materials are currently one of the most promising technical materials. Industrial sectors that use composites can be broadly listed as mechanical, electrical, transport or medical. They are characterised not only by light weight, high strength, and specific stiffness but also by toughness and fatigue strength. Moreover, they are often associated with corrosion, chemical and heat resistance, improved thermal insulation properties, or lower thermal expansion compared to conventional homogenous materials. A composite material can be defined as an efficient material system that is made by macroscopically combining two constituents - a reinforcement and a matrix. These two constituents do not dissolve or merge together and retain their individual properties. As a result, they act in unison to exhibit better engineering properties [1]. The disadvantage of composite materials is their higher production price and poor recyclability. Due to reinforcing fibers embedded in different directions, some composite materials have different mechanical properties, e.g. anisotropy. They may also react improperly to humidity and temperature change. Several manufacturing processes are regularly employed in the composites industry, such as rolling, hot pressing, or powder metallurgy and others. Technology most often used for the production of composite materials are additive manufacturing technologies, namely 3D printing. Currently, 3D printers are able to create composite materials and print the final product directly. However, 3D printing is realised in several ways. Individual technologies differ by the different embedding of layers, respectively by other parameters $[2,3]$.

Freeform Fabrication (FFF) is one of the modern ways of $3 \mathrm{D}$ printing. The principle is to gradually apply each layer by layer to the platform by using the extrusion head and nozzle. Each newly applied layer is bonded to the previous one due to the applied fiber being melted [4-6]. 3D printing can be influenced by setting various parameters, such as the number of layers, 3D printing structure (the angle of fiber embedding in the layer), layer 
thickness, inner infill shape and density, and others. These have less or more significant influence on the resulting physical or mechanical properties of final structural elements [7-9].

The paper deals with the impact of $3 \mathrm{D}$ printing settings on selected physical characteristics (mainly mechanical) of composite materials produced by additive FFF technology.

\section{Description of the problem}

The experimental part of the paper focuses on the research of selected mechanical characteristics of $3 \mathrm{D}$ printed composite structures based on Onyx material. Onyx is a material based on remarkably strong nylon in combination with carbon microfibers. Its physical properties are significantly better compared to conventional 3D printing polymers. In terms of mechanical properties, it is stronger, harder and, thanks to the carbon microfibers in the composite matrix itself, it is more suitable for the production of components subjected to multiaxial stress.

The observed properties of the presented printed structure, which we deal with, are obtained for subsequent modelling and simulation parameters. To be more specific, those parameters are Young's modulus, tensile strength, Poisson number and relative strains. The physical characteristics are investigated for two different 3D structures printed at $100 \%$ Onyx infill. The cause is to determine the effect of $3 \mathrm{D}$ printing on changing properties. The first case is so called " $\pm 45^{\circ}$ " $3 \mathrm{D}$ printing structure, where the individual fibers are embedded in the respective layer at angle of $+45^{\circ}$ and in the subsequent layer at angle $-45^{\circ}$. The second case is so called " $0{ }^{\circ} / 90^{\circ} " 3 \mathrm{D}$ printing structure. In this case, the individual fibers are embedded in the respective layer at angle of $0^{\circ}$ and in the subsequent layer at angle $90^{\circ}$. Given angles are related to the longitudinal axis of the printed part, which is equally oriented with the load direction. The fiber arrangement in the layer for printing structure of $\pm 45^{\circ}$ and $0^{\circ} / 90^{\circ}$ is shown in Figs. 1 and 2, respectively.

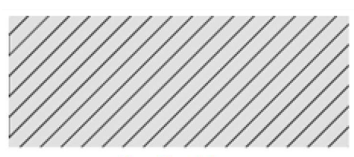

the first layer

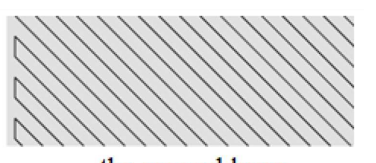

the second layer
Fig. 1. Scheme of first two layers for $\pm 45^{\circ}$ structure.

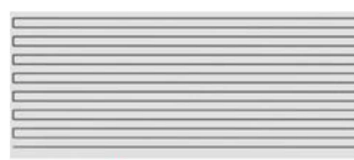

the first layer

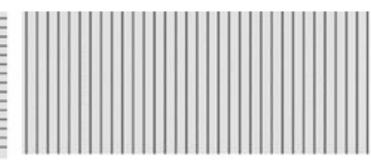

the second layer
Fig. 2. Scheme of first two layers for $0^{\circ} / 90^{\circ}$ structure.

\section{Experimental part}

Series of test samples made with the types of structures mentioned above have been subjected to an axial (tensile) test based on EN ISO 527-1 to identify the mechanical characteristics [10]. Samples were created by 3D printing on printer Mark Two. Figure 3 shows the testing specimen geometry. Figure 4 shows the experimental device used to perform the tests. Digital Image Correlation (DIC) technology was used to measure individual physical quantities in addition to the extensometer application approach [11-13]. DIC is an optical method based on the mutual correlation between individual images before and after the strain of the test sample. This method was used to measure the transverse and longitudinal strains. The measurement result is shown in Fig. 5, where it can be seen the course of the stress-strain dependence for both tested structures.

Based on the gained data, the dependence of the relative strain in the $y$-axis direction and the relative strain in the $x$-axis direction was identified for both tested structures (Fig. 6). The values for the relative strain in the $y$-axis direction are given in absolute value since they take negative values due to sample shrinking during the test. This graphical dependence has practical importance in determining the value of Poisson number, which is based on the ratio of the transverse $\left(\varepsilon_{y}\right)$ and the longitudinal strain $\left(\varepsilon_{x}\right)$.

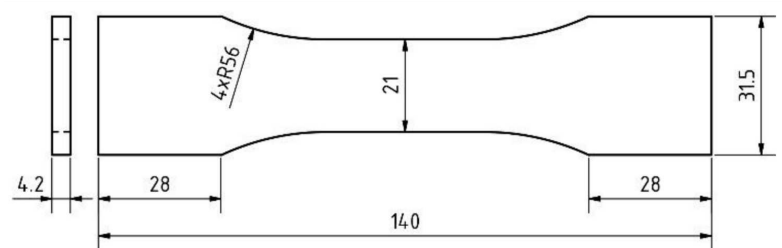

Fig. 3. Scheme of specimen geometry.

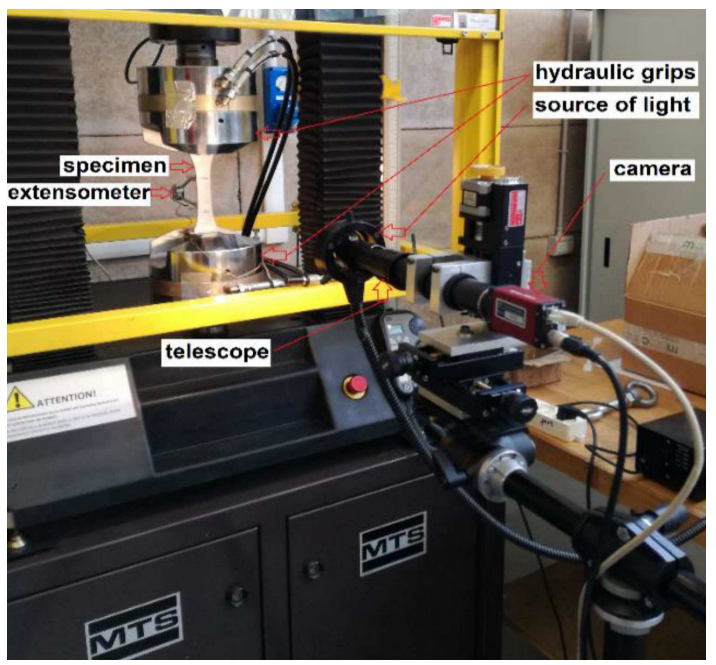

Fig. 4. Experimental setup and its elements. 


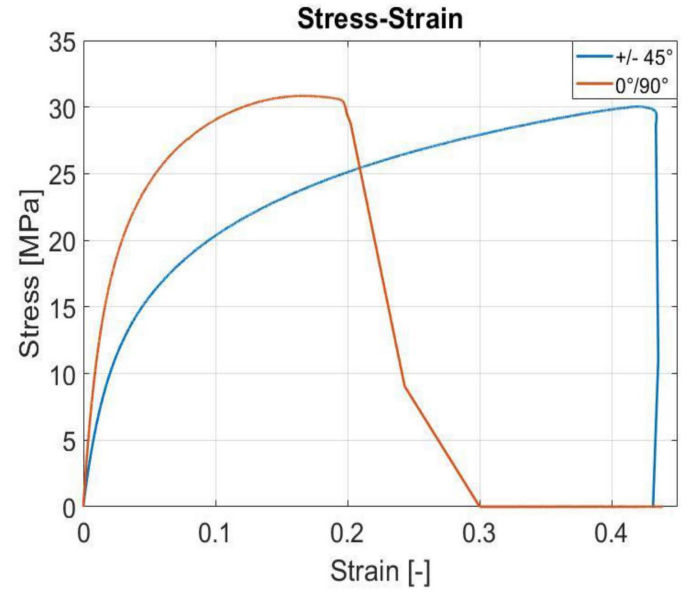

Fig. 5. Stress-Strain graph for $\pm 45^{\circ}$ and $0^{\circ} / 90^{\circ}$ structure.

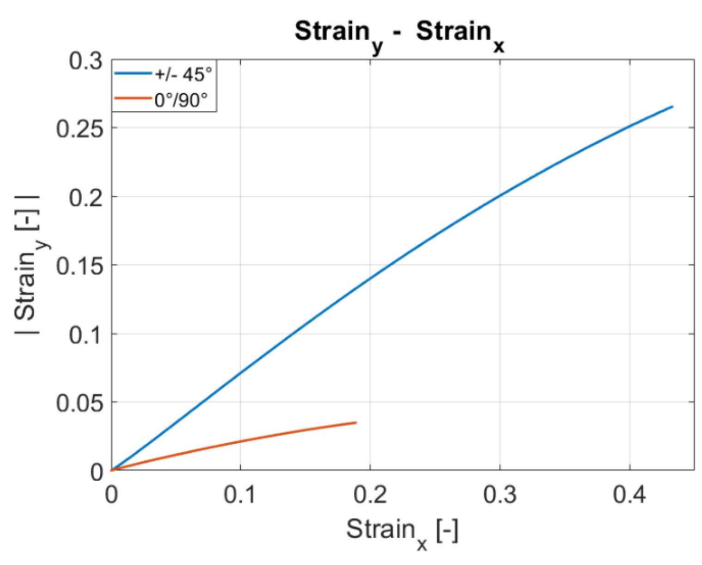

Fig. 6. Strain $\varepsilon_{y}$ vs. Strain $\varepsilon_{x}$ behaviour for $\pm 45^{\circ}$ and $0^{\circ} / 90^{\circ}$ structure.

Table I shows the values of the monitored parameters for individual structures of the test samples.

The experimental value of Poisson number for $\pm 45^{\circ}$ structure was consequently verified based on the parameters gained from the measurements. The following equations apply [14]:

$$
\begin{aligned}
\frac{1}{E_{x}} & =\frac{\cos ^{4}(\theta)}{E_{11}}+\frac{\sin ^{4}(\theta)}{E_{22}} \\
+ & \left(\frac{1}{G_{12}}-\frac{2 \mu_{12}}{E_{11}}\right) \cos ^{2}(\theta) \sin ^{2}(\theta), \\
\mu_{x y} & =E_{x}\left[\frac{\mu_{12}}{E_{1}}-\cos ^{2}(\theta) \sin ^{2}(\theta)\right. \\
& \left.\times\left(\frac{1}{E_{1}}+\frac{2 \mu_{12}}{E_{1}}+\frac{1}{E_{2}}-\frac{1}{G_{12}}\right)\right]
\end{aligned}
$$

where $E_{x}$ is Young's tensile modulus for $\pm 45^{\circ}$, $E_{11}=E_{22}$ is Young's tensile modulus for $0^{\circ} / 90^{\circ}$, $\mu_{12}$ is Poisson number for structure $0^{\circ} / 90^{\circ}, \mu_{x y}$ is Poisson number for structure $\pm 45^{\circ}, G_{12}$ is shear modulus for the structure $0^{\circ} / 90^{\circ}, 0^{\circ}$ is the angle of fiber embedding in the layer, in our case $45^{\circ}$. Substituting the known quantities into (1) with its
TABLE I

Measured parameters for $\pm 45^{\circ}$ and $0^{\circ} / 90^{\circ}$ structure.

\begin{tabular}{l|c|c}
\hline \hline \multicolumn{1}{c|}{ Structure } & $\pm 45^{\circ}$ & $0^{\circ} / 90^{\circ}$ \\
\hline tensile modulus $[\mathrm{MPa}]$ & 604 & 1154 \\
Poisson's ratio & 0.65 & 0.25 \\
tensile stress at break & 30 & 30.9 \\
tensile strain at break & 0.42 & 0.17
\end{tabular}

TABLE II

Experimental and mathematical value of Poisson's ratio.

\begin{tabular}{c|c|c|c}
\hline \hline Structure & $\mu_{\text {exp. }}$ & $\mu_{\text {mat. }}$ & Deviation \\
\hline $\pm 45^{\circ}$ & 0.65 & 0.61 & $6 \%$
\end{tabular}

subsequent simplification, the parameter $G_{12}$ can be calculated and further substituted for (2) to calculate $\mu_{x y}$. The value of Poisson number for $\pm 45^{\circ}$ printing structure, which was experimentally gained and mathematically calculated, is shown in Table II.

\section{Results}

The results from the experimental measurements and the values given by the technology submitter for $+/-45^{\circ}$ structure can be found in Table III and for $0^{\circ} / 90^{\circ}$ structure in Table IV. These data also show deviations of measured values for individual structures from standard values given by the technology submitter.

Based on the evaluation of experimental measurements in order to identify selected mechanical properties, it is possible to state the significant influence of $3 \mathrm{D}$ printing on some of the mentioned mechanical properties. The value of standard Young's modulus for pure Onyx is $1400 \mathrm{MPa}$. By the experiment was found out that the value of Young's modulus for $\pm 45^{\circ}$ structure is $604 \mathrm{MPa}$, and the value for $0^{\circ} / 90^{\circ}$ structure is $1154 \mathrm{MPa}$. Based on these findings, it is possible to state that under the influence of 3D printing, Young's modulus changes notably. There is a significant decrease up to approximately $57 \%$ with $\pm 45^{\circ}$ structure compared to the values reported by the technology submitter. For $0^{\circ} / 90^{\circ}$ structure, Young's modulus decreases slightly by approximately $18 \%$.

The tensile strength for individual structures does not change significantly and remains at the value of about $30 \mathrm{MPa}$. Thus, it can be stated that the structure change of $3 \mathrm{D}$ printing does not have a significant impact on the tensile strength of the printed components. The tensile stress at break for the default value of Onyx is 0.58 . For $\pm 45^{\circ}$ structure, the tensile strain at break was experimentally measured, and the value was 0.42 . The value for $0^{\circ} / 90^{\circ}$ structure was 0.17 . Again, the results show that the structure of $3 \mathrm{D}$ printing significantly 
TABLE III

Comparison of measured values for $\pm 45^{\circ}$ structure.

\begin{tabular}{l|c|c|c}
\hline \hline \multicolumn{1}{c|}{ Structure/Material } & Onyx & $\pm 45^{\circ}$ & Deviation \\
\hline tensile stress at break [MPa] & 30 & 30 & $0 \%$ \\
tensile modulus [MPa] & 1400 & 604 & $57 \%$ \\
Poisson ratio & - & 0.65 & - \\
tensile strain at break & 0.58 & 0.42 & $28 \%$
\end{tabular}

TABLE IV

Comparison of measured values for $0^{\circ} / 90^{\circ}$ structure.

\begin{tabular}{l|c|c|c}
\hline \hline \multicolumn{1}{c|}{ Structure/Material } & Onyx & $0 / 90^{\circ}$ & Deviation \\
\hline tensile stress at break [MPa] & 30 & 30.9 & $3 \%$ \\
tensile modulus [MPa] & 1400 & 1154 & $18 \%$ \\
Poisson ratio & - & 0.25 & - \\
tensile strain at break & 0.58 & 0.17 & $71 \%$
\end{tabular}

affects the value of the strain at break. For $\pm 45^{\circ}$ structure the tensile strain decreases by $28 \%$, and for $0^{\circ} / 90^{\circ}$ structure, it significantly decreases up to $71 \%$ compared to the known values.

The technology submitter does not provide Poisson number for Onyx material. Therefore, the values of Poisson number for $\pm 45^{\circ}$ and $0^{\circ} / 90^{\circ} 3 \mathrm{D}$ printing structures were found by experimental measurements. To be more specific, for $\pm 45^{\circ}$ structure, the value of Poisson number was 0.65 , and the value for $0^{\circ} / 90^{\circ}$ structure was 0.25 . Based on the experiments, one can claimed that the structure of 3D printing also has a significant influence on the value of Poisson number. The difference in Poisson number between $\pm 45^{\circ}$ and $0^{\circ} / 90^{\circ}$ is $\approx 62 \%$. With the use of (1) and (2), Poisson number for the $\pm 45^{\circ}$ structure was mathematically verified.

\section{Conclusions}

Experimental testing confirmed the significant impact on some of the physical parameters if the 3D printed structure is changed. It has been verified that the 3D print structure of Onyx material can affect the tensile strain at break of the sample more than double. An important finding is that the different structure of 3D printing is seen in the increasing value of Poisson number to the level above 0.5. The 3D printing structure also has a significant impact on Young's tensile modulus, where differences $(50 \%)$ were measured compared to conventionally produced samples.
The knowledge gained has a significant influence on the design practice in connection with $3 \mathrm{D}$ printing of machine components. This gained knowledge is suitable to apply in the creation of mathematical models based on the finite element method.

\section{Acknowledgments}

This work has been supported by grant agency VEGA project No. 1/0073/19.

\section{References}

[1] M.K. Buragohain, Composite Structures (CRC Press, Boca Raton, 731 p., 2017).

[2] A. Vaško, J. Belan, Mater. Today Proc. 4, 5985 (2017).

[3] F. Bárnik, M. Vaško, M. Sága, M. Handrik, A. Sapietova, MATEC Web Conf. 254, 010118 (2019).

[4] R. Halama, A. Markopulos, F. Fojtik, J. Famfulík, Mater. Sci. Eng. Technol. 48, 814 (2017).

[5] L.G. Blok, M.L. Longana, H. Yu, B.K.S. Woods, Addit. Manuf. 22, 176 (2018).

[6] R.T.L. Ferreira, I.C. Amatte, T.A. Dutra, D. Bü rger, Compos. Part B: Eng. 124, 88 (2017).

[7] K. Gnanasekaran, T. Heijmans, S. van Bennekom, H. Woldhuis, S. Wijnia, D. de With, H. Friedrich, Appl. Mater. Today 9, 21 (2017).

[8] M. Ivey, G.W. Melenka, J.P. Carey, C. Ayranci, Adv. Manuf.: Polym. Compos. Sci. 3, 81 (2017).

[9] T. Letcher, M. Waytashek, in: Proc. Int. Mechanical Engineering Congress \&3 Exposition Vol. A2, 2014.

[10] EN ISO 527-1 "Plastics - Determination of Tensile Properties — Part 1: General Principles".

[11] B. Pan, K. Qian, H. Xie, A. Asundi. Meas. Sci. Technol. 20, 062001 (2009).

[12] T.S. Smith, B.K. Bay, M.M. Rashid, Exp. Mech. 42, 272 (2002).

[13] B. Pann, Appl. Opt.48, 1535 (2009).

[14] A. Bernasconi, M.D. Kharshiduzzaman, L. Comoli, J. Adhesion 92, 440 (2016). 